\title{
NUMERICAL COMPUTATION OF THE ELECTROMAGNETIC BIAS IN GNSS-R ALTIMETRY
}

\author{
Ali Ghavidel ${ }^{1}$, Student Member IEEE, Domenico Schiavulli ${ }^{2}$, Student Member IEEE,
} Adriano Camps ${ }^{1}$, IEEE Fellow

Email: ali.ghavidel@tsc.upc.edu,domenico.schiavulli@uniparthenope.it, camps@tsc.upc.edu

\begin{abstract}
In radar altimetry the electromagnetic (EM) bias is originated by the smaller reflectivity of wave crests than troughs, thus the average sea surface height is under-estimated. Bias uncertainty is currently the largest factor in altimetry error budgets. The EM bias in a bistatic forward-scattering configuration at L-band, such as in Global Navigation Satellite SystemsReflectometry (GNSS-R) altimetry, remains one of the major sources of uncertainty in the altimetry error budget. In this work the EM bias is computed using numerical simulations. To do so, a time-dependent synthetic non-Gaussian sea surface is created using the PiersonMoskowitz and Elfouhaily sea surface height spectra and spreading function. The sea surface is then discretized in facets and "illuminated" using a Right Hand Circular Polarization (RHCP) GNSS signal, previously recorded by an up-looking antenna connected to a data logger. The waves scattered from each facet are then computed using the Physical Optics (PO) method under the Kirchhoff Approximation (KA), and the radar cross-section of each facet is computed. The scattered electric fields are "collected" by a down-looking Left Hand Circular Polarization (LHCP) antenna, and the electromagnetic bias (EM bias) is computed based on its fundamental definition. The numerical model is validated against Millet's model (a combined model of the Weakly Non-Linear and Modulation Transfer models) with real data at $\mathrm{C}$ - and Ku-bands. Then, the numerical model is applied at L-band, for bistatic configurations, including different azimuth angles, and different wind speeds. It is found that the EM bias is almost insensitive to the sea surface spectra selected and increases with increasing wind speed and incidence/scattering angle (up to $\sim 20 \mathrm{~cm}$ at $\theta_{i, s}=45^{\circ}$ and $U_{10}=12 \mathrm{~m} / \mathrm{s}$ ), and it also exhibits a non-negligible azimuthal dependence, that must be accounted for in the error budgets of upcoming GNSS-R altimetry missions.
\end{abstract}

Index Terms electromagnetic bias, GNSS-R, ocean altimetry, scattering.

\footnotetext{
${ }^{1}$ Universitat Politecnica de Catalunya-BarcelonaTech, and IEEC/CTE-UPC. Campus Nord, D3, 08034, Barcelona, Spain.

${ }^{2}$ Università degli Studi di Napoli, "Parthenope", Italy.
} 


\section{INTRODUCTION}

Satellite altimeters measure the distance from the satellite to the sea surface by measuring the round-trip time of a radar pulse, typically in a nadir-looking configuration. The amplitude and shape of the so-called waveform (cross-correlation of the signal received by a downlooking antenna and the locally-generated replica of the transmitted signal) include information of the characteristics of the surface where the scattering takes place. Conventional radar altimeters use a nadir-looking configuration, high transmitted power, large bandwidth, and high carrier frequency to be less sensitive to ionospheric effects. On the other hand, upcoming GNSS-R altimeters use a bistatic (off-nadir) configuration, much lower transmitted power, narrower bandwidth ${ }^{3}$, and use frequency bands allocated for radionavigation ${ }^{4}$.

As compared to conventional radar altimeters, these limitations translate into a poorer height resolution $\left(\sigma_{\mathrm{h}}=20-30 \mathrm{~cm}_{\mathrm{rms}}\right)$, although the spatio-temporal sampling is maximized by receiving reflected navigation signals from a wide range of incidence angles, typically up to $35^{\circ}$ [1]. Usually, a GNSS-R receiver in a Low Earth Orbit (LEO) can track more than 10 different GPS reflections at the same time, therefore providing a much larger swath, and a much more reduced revisit time. This concept is illustrated in Fig. 1, where a space-borne GNSS-Reflectometer is receiving multiple scattered signals from different navigation satellites and constellations. In conventional GNSS-R only the reflected signal has to be collected, which is then cross-correlated with a locally generated replica of the transmitted one, while in interferometric GNSS-R both the direct and the reflected signals are collected, which is then cross-correlated among them.

\footnotetext{
${ }^{3}$ In conventional GNSS-R (cGNSS-R) the bandwidth is limited to the bandwidth of the publicly available codes (e.g. $2 \mathrm{MHz}$ for the GPS L1 C/A code). In interferometric GNSS-R (iGNSS-R) the bandwidth is limited to the bandwidth of the whole navigation signals (e.g. 25 MHz for the GPS composite signal resulting from the combination of the C/A, P and M codes), or up to $51 \mathrm{MHz}$ if the E5 AltBOC signal is used. However, while the altimetry accuracy depends on the bandwidth, the EM bias does not. Note: C/A stands for Coarse/Acquisition, $\mathrm{P}$ for Precise, and $\mathrm{M}$ for Military pseudo-random noise (PRN) codes.

${ }^{4}$ Typically L1 (1575.420 MHz) and L2 (1227.600 MHz), or L1 and L5 (1176.450 MHz), in order to compensate for ionospheric effects.
} 


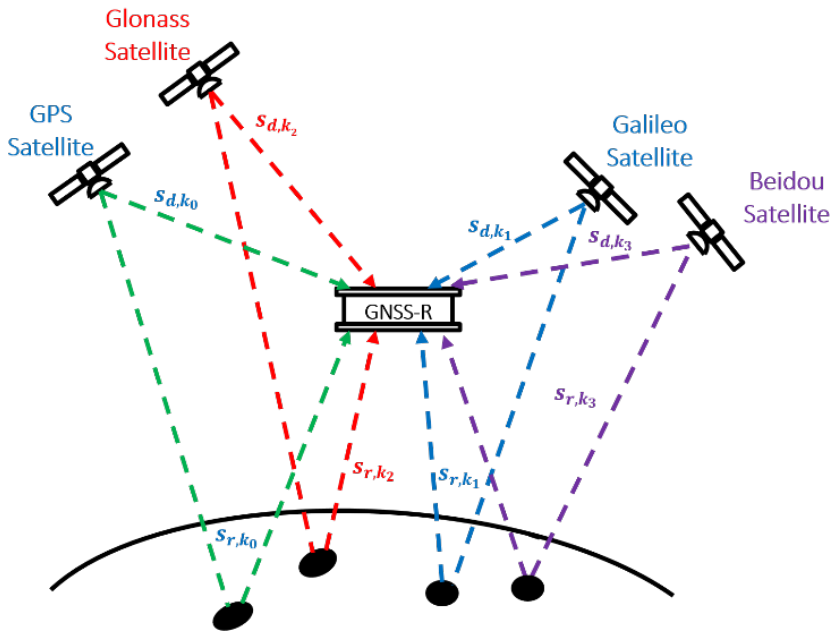

Fig. 1. GNSS-R concept.

At the same time, GNSS-R instruments can also be used to infer other ocean surface properties such as the sea state (significant wave height), and the wind speed by comparing the shape and amplitude of the waveform of the reflected signal to that of the direct one [2]. In a GNSS-R instrument, the observable is obtained by the complex cross-correlation of the scattered signals either with a locally-generated replica of the transmitted signal (conventional GNSS-R or cGNSS-R), or with the direct down-looking signal (interferometric GNSS-R or iGNSS-R) during the coherent integration, followed by an incoherent integration to reduce speckle noise. The time delay of the scattered wave can be estimated from the delay of the maximum derivative of the waveform [3].

In addition to the geometric delay to be measured, there are several other contributions to the estimated average delay: the clock offsets of receiver and transmitter relative to GPS time scale, and the tropospheric, and the ionospheric delays. The tropospheric dry delay has an average value of $2.3 \mathrm{~m}$, and a residual error of $\sim 0.7 \mathrm{~cm}$, and the tropospheric wet delay is highly variable, typically from 5 to $30 \mathrm{~cm}$, and it is computed using atmospheric models or microwave radiometers, with a precision of $\sim 1.1 \mathrm{~cm}$. The ionospheric delay is also highly variable, typically from 1 to $20 \mathrm{~m}$, and it is computed using dual-frequency observations with a precision of $\sim 0.5 \mathrm{~cm}$.

In [4] a detailed altimetry error budget for an iGNSS-R instrument was performed to assess the rms altimetry precision. The main results are summarized in Table 1. 
Table 1. Instrument performance for a PARIS IoD - like instrument at levels 1 (range precision) and 2 (height precision) including ionospheric corrections for GPS and Galileo at $\theta_{i}=0^{\circ}$ and $35^{\circ}$, assuming 1 ms coherent integration time, 14500 incoherent averages, and typical satellite transmitted powers (modified from [4]).

\begin{tabular}{lcc}
\hline Altimetry precision [cm $\left.\mathrm{cms}_{\mathrm{rms}}\right]$ & $\sigma_{\mathrm{h}} @ \theta_{\mathrm{i}}=35^{\circ}$ & $\sigma_{\mathrm{h}} @ \theta_{\mathrm{i}}=0^{\circ}$ \\
\hline Level - 1 Lower band (L5+E5) & L5: 56.0 & L5: 29.7 \\
LB $=1186.6 \mathrm{MHz}$ & E5: 15.5 & E5: 8.3 \\
Level 1 - Higher band (L1 + E1) & L1: 37.2 & L1: 16.4 \\
LH $=1575.42 \mathrm{MHz}$ & E1: 26.6 & E1: 12.8 \\
Level 2 & L1\&L5: 60.5 & L1\&L5: 30.5 \\
(LB + LH + ionospheric corrections) & E1\&E5: 27.7 & E1\&E5: 13.7 \\
\hline
\end{tabular}

As it will be shown, the estimated EM bias is on the order of magnitude of the expected rms altimetry precision.

In order to perform a study as realistic as possible, a true direct (multipath-free) GPS signal was first recorded to simulate the signal illuminating a synthetic sea surface. The scattered wave is then computed for each facet using the Physical Optics (PO) method under the Kirchhoff Approximation (KA) because of its higher accuracy [5] as compared to the Geometric Optics [6]. The scattered signal is then used to compute the radar cross-section density $\left(\sigma^{0}\right)$ to be used in Eqn. (1).

In conventional satellite altimetry, the EM bias is one of the most difficult errors to compensate for, and it requires models to be compensated for. The EM bias was first reported in [7]. A number of studies on the EM bias have been performed so far for nadir-looking, and small off-nadir angles, but because of its significance, it is still a matter of research. In general, there are two approaches to estimate the EM bias: the Weakly Non-Linear (WNL) theory [8], and the Modulation Transfer Function (MTF) model [9]. Jackson [10] applied the WNL theory to estimate the EM bias in one-dimension. In the reflection of radar pulses from the sea surface at near-vertical incidence angles, non-Gaussian ocean wave statistics were accounted for using the joint probability density function (PDF) of the surface's height and slope computed using the Longuet-Higgins theory [11]. In addition to the sea surface height, the nature of the altimeter's response to a rough sea surface allows to infer also other parameters such as the significant wave height, the wind speed, while the backscatter 
coefficient or return power can also be used to determine topographic changes over ice sheets, lakes, rivers, and deserts, or to estimate ice and snow thickness [11-14].

The estimation of the EM bias for a two-dimensional surface using the WNL theory was proposed in $[15,16]$, although it is applicable only for long waves. To overcome the previous limitations, in [8] a modified WNL theory was proposed to estimate the EM bias applying a unified directional sea surface spectrum that was able to account for long and short waves [17].

Later, an analytical model to compute the EM bias was also studied based on the twodimensional hydrodynamic modulation [9]. The strong point of the hydrodynamic modulation theory relies on the linearization of the wave action balance equation. This linearization yields to the so-called modulation transfer function or MTF. In the Fourier domain, the MTF is a function of both the long and the short wave-numbers, and it has also been used to estimate the EM bias in two-dimensional surfaces [18].

The EM bias for a monostatic configuration and for small off-nadir incidence angles was considered in [19, 20], and more recently in [21, 22] using a combination of the WNL and MTF models. In [21] a theoretical formula was proposed that showed that the EM bias depends on the incidence angle, and it was demonstrated experimentally.

Recently, a Monte-Carlo study on the EM bias has been performed using non-linear numerical hydrodynamic simulations [23], and applying the KA PO method to simulate the scattered signal [24]. This work showed a non-negligible impact of the short waves on the EM bias for different frequencies, and a nadir-looking configuration in a one-dimensional scale.

In this work, in order to assess the impact of the particular sea surface spectra, the PiersonMoskowitz [25] and the Elfouhaily et al. [26] spectra, are used to generate a synthetic twodimensional sea surface, including the spreading function (the up-wind and cross-wind asymmetry) [27]. The non-linearity of the generated sea surface is assessed in [28]. The surface is illuminated by a GPS signal, the scattered wave is computed using KA PO method, from which the scattering cross-section is estimated [21, 22, 29, 30]. 
The above mentioned method (combined WNL and MTF) has been implemented and validated with higher frequency data (C- and Ku-bands), first (Fig. 6). Then, the method is applied to the calculation of the EM bias at L-band for off-nadir incidence angles such as in GNSS-R systems (Fig. 7), and the results at L- and Ku-bands are satisfactorily compared to the numerical model proposed in this work (Fig. 10). Once the numerical model is validated, the obtained EM bias is computed as a function of the wind speed, incidence/scattering, and azimuth angles. The proposed method is numerically efficient and stable, and because of the large spatial averaging, it only requires a modest number (10) of Monte-Carlo simulations to provide statistically meaningful results.

This work is organized as follows. Section 2 gives the background for the EM bias computation, the generation of the non-Gaussian sea surface, and the illumination of the generated sea surface by a RHCP (GPS-like) electromagnetic wave, and the computation of the scattered wave using the KA-PO. In Section 3, the off-nadir EM bias combined model (WNL+MTF) is validated at C- and Ku-bands, and then applied at L-band. Section 4 presents the results of the proposed numerical method, which are validated against the combined model, and discusses them as a function of the sea surface spectra model, frequency band (LC-, and Ku-band), surface discretization, wind speed, incidence/scattering and azimuth angles. Finally, Section 5 summarizes the main conclusions.

\section{EM BIAS COMPUTATION}

In order to estimate the EM bias, three main simulator blocks have to be implemented:

1) the generation of a time-dependent non-Gaussian realistic sea surface,

2) the generation of the RHCP direct signal (in this case an actual GNSS signal collected using an up-looking choke-ring antenna mounted on the tip of a mast in the roof of the building connected to a data logger) that illuminates the sea surface, and

3) the computation of the scattered signal using, for example, the KA-PO method [5, 21, 22, 29]. This method has proven to be quite accurate for forward scattering even for polarimetric studies.

These steps are considered separately in the next sections, after reviewing the existing methods to compute the EM bias. 


\subsection{Summary of existing analytical methods to compute the EM bias}

There are three main methods to estimate the EM bias: the Weakly Non-Linear (WNL) theory [8], the Modulation Transfer Function (MTF) [9], and a combined model (WNL \& MTF).

1. The EM bias was first estimated using an improved WNL theory, which involved both long and short waves effects. The impact of long waves is a modulation of the radar crosssection density $\left(\sigma^{0}\right)$ by the waves' slopes, which is actually correlated to the surface's elevation, because of the non-linear processes caused by the wave-wave interactions. The coupling effect between long and short waves is also accounted for in the EM bias computation [8, 10, 15, 16, 24].

2. The MTF model was used to estimate the EM bias on the two-scale hydrodynamic assumption [31]. The MTF separates the long and short waves spectrum, which shows separately the impact of both the long and the short waves on the EM bias.

3. In GNSS-R, the incidence angle may be quite large $\left(35^{\circ}-45^{\circ}\right)$, and its impact has to be considered. Then, a suitable method to estimate the EM bias has to be derived. A combined method that included the improved WNL and the MTF model, was implemented by Millet et al. [21, 22] to estimate the EM bias at Ku-band. In this method, the impact of long waves is computed as the contribution of the improved WNL theory, while the impact of short waves is accounted for using the MTF model.

The combined model (WNL + MTF) is validated with real data and it is considered in this work as a reference to estimate the off-nadir EM bias at other frequencies and geometries, accounting for the long and short waves EM bias contributions separately [32-37].

In [21], the off-nadir EM bias model was computed as a function of the long wave surface statistics (Gram-Charlier series [15]), and modified by the small wave coefficient. The small wave coefficient was computed from the short wave surface scattering obtained using the PO method. The incidence angle was included in the computation of the small wave scattering [21] (Appendix A).

In the off-nadir EM bias model, the composite surface scattering model used a cut-off wavenumber $k_{\text {sep }}$ to divide the surface into long and short wave scales. Because of the weak sensitivity to $k_{s e p}$, the unified Elfouhaily' spectrum was considered. For short wave scales, 
the significant slope of spectrum was used to separate the wavelengths over a wide range of wind speeds [21, 32, 33].

This combined EM bias model is first validated at Ku-band (14 GHz), and C-band (5.2 GHz). It is then extrapolated at L-band (1.575 GHz) for GNSS-R applications, and used for intercomparison with (validation of) the numerical results obtained later. Results are presented in Section 3.

\subsection{Numerical Computation of the EM bias}

The EM bias basic definition is simply the ratio of the average of the radar cross-section density $\left(\sigma^{0}\right)$ times the sea surface elevation $(\xi)$, divided by the average $\sigma^{0}[17]$ :

$$
\beta_{E M}=\frac{\left\langle\xi \sigma^{0}\right\rangle}{\left\langle\sigma^{0}\right\rangle}
$$

To evaluate Eqn. (1), the surface wave height and $\sigma^{0}$ are required. The sea surface wave height and orientation for each facet are known, since they are the outputs of the sea surface generator (Section 2.2.1). The value of $\sigma^{0}$ is computed using the KA PO method (Section 2.2.3).

\subsubsection{Generation of a synthetic non-Gaussian sea surface}

The assessment of the average EM bias [14] cannot consider the ocean surface as a Gaussian surface. As a matter of fact, wave crests are more peaked than the wave troughs, and this effect translates into a surface height PDF with a non-symmetric behavior, that has a nonzero skewness coefficient [25]. There are several methods to generate a non-Gaussian surface (e.g. [23]), with benefits and drawbacks (mainly the large computational time), that have been investigated in [38].

In this work, two directional sea surface height spectra (Pierson-Moskowitz and Elfouhaily) are selected, and then converted to directional spectra using a cosine-shape, and a unified spreading model function, respectively [25, 26]. In order to assess the non-Gaussianity of the generated surface, the numerical method proposed in [28] is applied here.

A snapshot of the non-Gaussian sea surface is shown in Fig. 2, using the parameters listed in Table 2. 
Table. 2. Parameters used to synthesize the (non-Gaussian) time-domain sea surface.

\begin{tabular}{ll}
\hline Parameter & Value/Unit \\
\hline Patch area & $1000 \times 1000 \mathrm{~m}^{2}$ \\
Wind Speed & $5 \mathrm{~m} / \mathrm{s}$ \\
Wind Direction & $45^{\circ}$ \\
Sea Surface Spectrum & Elfouhaily [26] \\
Anisotropic Spectrum & Unified Spreading Function \\
& (up-wind/cross-wind asymmetry) \\
\hline
\end{tabular}

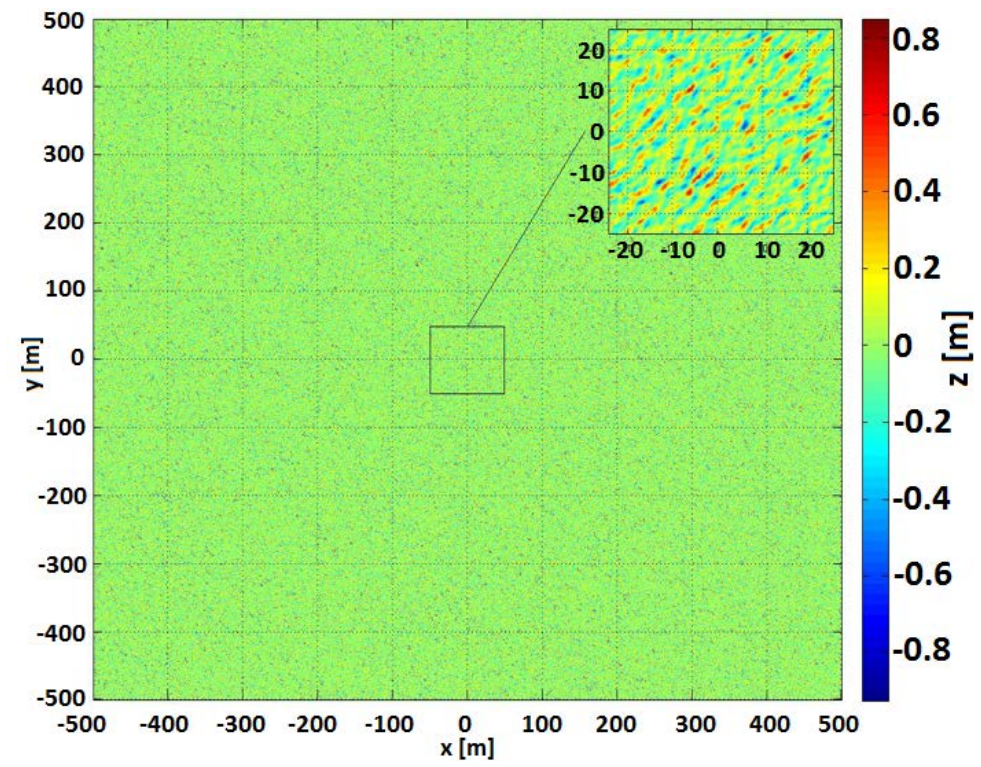

Fig. 2. Snapshot of the non-Gaussian sea surface generated using Elfouhaily's spectrum for a wind speed $=5 \mathrm{~m} / \mathrm{s}$, and wind direction $\phi=45^{\circ}$. Upper right corner: zoom of the central part to better appreciate the wavy structure. Color bar indicates the sea surface height of each pixel.

Finally, the non-Gaussian surface height PDF is obtained for three different wind speeds 5, 10, $15 \mathrm{~m} / \mathrm{s}$, and it is presented in Fig. 3. The estimated PDF's are similar to the Edge-Worth expansion, demonstrated experimentally, and theoretically in [28, 39-46], and deviate from the Gaussian model as the wind speed increases. 

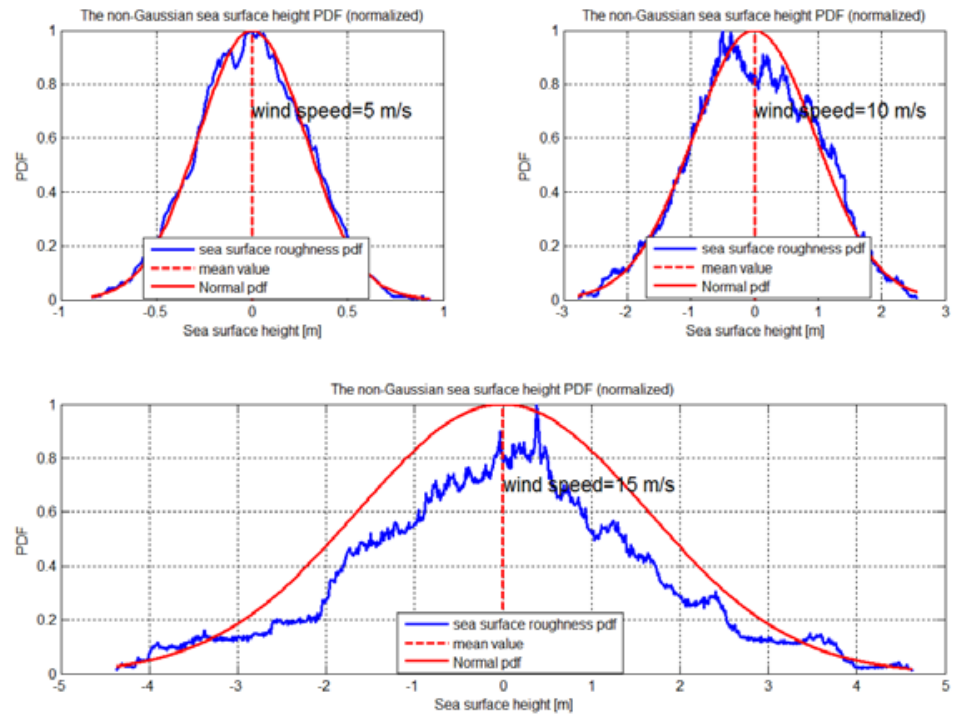

Fig. 3. Computed sea surface height PDF (normalized) from 10 realizations for different wind speeds $(5,10,15 \mathrm{~m} / \mathrm{s})$. Note that the PDF departs from Gaussian as wind speed increases.

\subsubsection{Generation of the direct signal}

GPS satellites transmit RHCP waves $^{5}$ at L1 $(1575,42 \mathrm{MHz})$, modulated using the Coarse/Acquisition (C/A) code for civilian applications. In order to be more realistic, a direct multipath-free GPS signal was recorded using a GPS antenna, an L-band microwave amplifier, and a data logger.

\subsubsection{Computing the scattered wave using the Kirchhoff Approximation under the scalar approximation (Physical Optics -PO- method)}

The KA PO method has been implemented in the forward scattering scenario to estimate the EM bias [21, 22, 29, 30]. Once the time domain sea surface (Appendix B) is illuminated, the instantaneous scattered field is computed for each facet in which the three-dimensional surface is discretized. Each point of the sea surface is described by its displacement with respect to the flat surface (height), a unit normal vector $\widehat{\boldsymbol{n}_{l}}$ perpendicular to each facet, where the tilting angle from the $\hat{Z}$ axis is given by $\theta=\arccos \left(\hat{Z} . \widehat{n_{l}}\right)$, and the rotation angle $\varphi$ (Fig. 4).

${ }^{5}$ The residual left-hand circularly polarized (LHCP) transmitted signal is neglected in this study. 
In addition, a finer sea surface discretization is applied to study the impact of short-waves on the radar cross-section. The scattered wave then is computed by summing up coherently all the contributions from all facets (Appendix C).

The basics of the KA model assumes that locally the surface can be approximated by an inclined plane. The surface correlation length must be larger than electromagnetic wavelength, and the standard deviation of the surface height must be small, so that the average radius of curvature is much larger than the electromagnetic wavelength [5, 29].

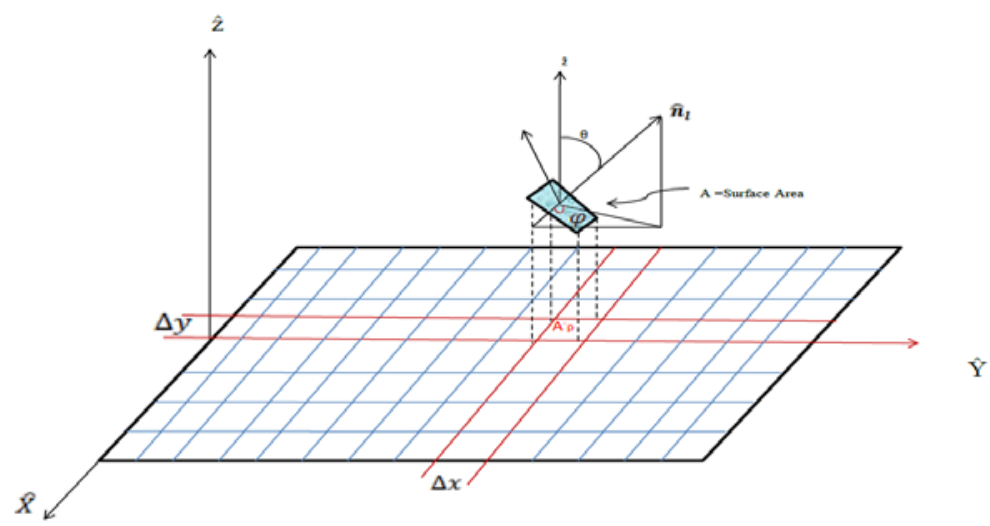

Fig. 4. Representation of the surface facet discretization.

It has been proven that, at L-band, in a forward scattering scenario ${ }^{6}$, cross-polar terms can be also accurately described using the KA PO method [5, 29]. The facet size height and orientation are discussed (Appendix C), and the facet surface is assumed as non-metallic (dielectric constant $\varepsilon_{r}=73+j 57.5$ ) [47]. In this study, the generated sea surface is discretized into facets of an equivalent size close to the electromagnetic wavelength $(\sim 20 \mathrm{~cm})$. The validation of this approach is assessed in Section 4.2.

This procedure is implemented over a square synthetic sea surface of $6 \mathrm{~km}$ side, much larger than the electromagnetic wavelength, and then the wavelength of the sea waves, even for strong winds and developed seas. For computational purposes, the surface was divided in blocks of $1000 \mathrm{~m} \times 1000 \mathrm{~m}$ each one, discretized in $25 \times 10^{6}$ points, for which each of the 10 Monte Carlo realizations takes about 27 min.

\footnotetext{
${ }^{6}$ The scattered wave is mostly LHCP for an incident wave at RHCP.
} 


\section{Validating the off-nadir EM bias combined model}

To validate the combined model with the few existing data (scatter plot in Fig. 6 in terms of the significant wave height), the relationship between the significant wave-height and the wind speed was obtained first using Elfouhaily et al.'s spectrum (Fig. 5). This relationship is applied to estimate in the off-nadir EM bias using the combined model at C- $(5.2 \mathrm{GHz})$ and $\mathrm{Ku}-(14 \mathrm{GHz})$ bands, and to compare these results with the few existing experimental data [21] (Fig. 6). The agreement between the simulation results and the experimental data is pretty good. Once the implementation of the combined model is validated, the EM bias at Lband $(1.575 \mathrm{GHz})$ can be computed. Results are presented in Fig. 7, now in terms of the wind speed. At $\sim 12 \mathrm{~m} / \mathrm{s}$, the EM bias increases with decreasing frequencies: $\sim 12 \mathrm{~cm}$ at Ku-band, $\sim-17 \mathrm{~cm}$ at C-band, and $\sim-19 \mathrm{~cm}$ at L-band.

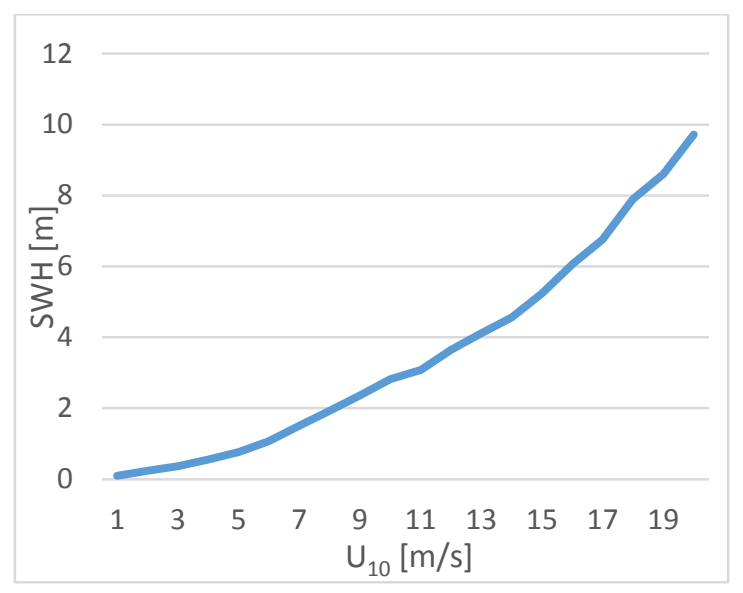

Fig. 5. Significant wave-height vs. wind speed computed using Elfouhaily’s spectrum [26]. 


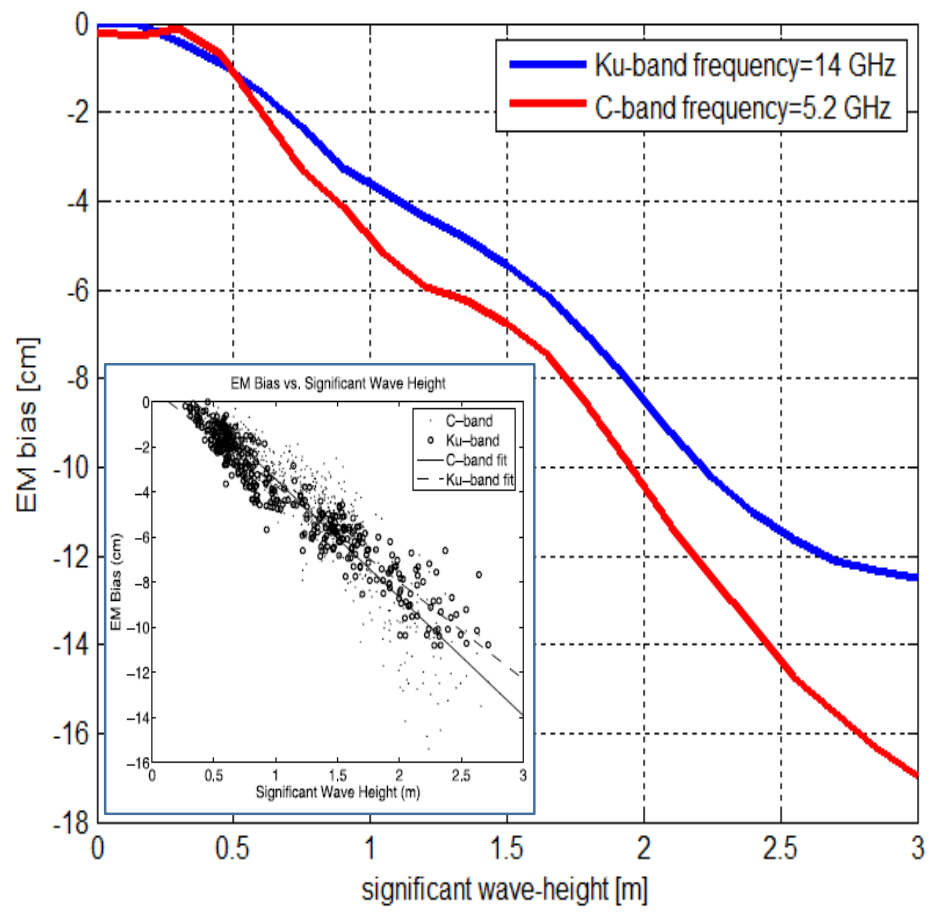

Fig. 6. The EM bias computed $\left(\theta=0^{\circ}\right)$ at C-and Ku-band using the combined model, and validation with existing experimental data (scatter plot in subplot on the low left corner from [21, Fig. 8, Eqns. 14, 15]).

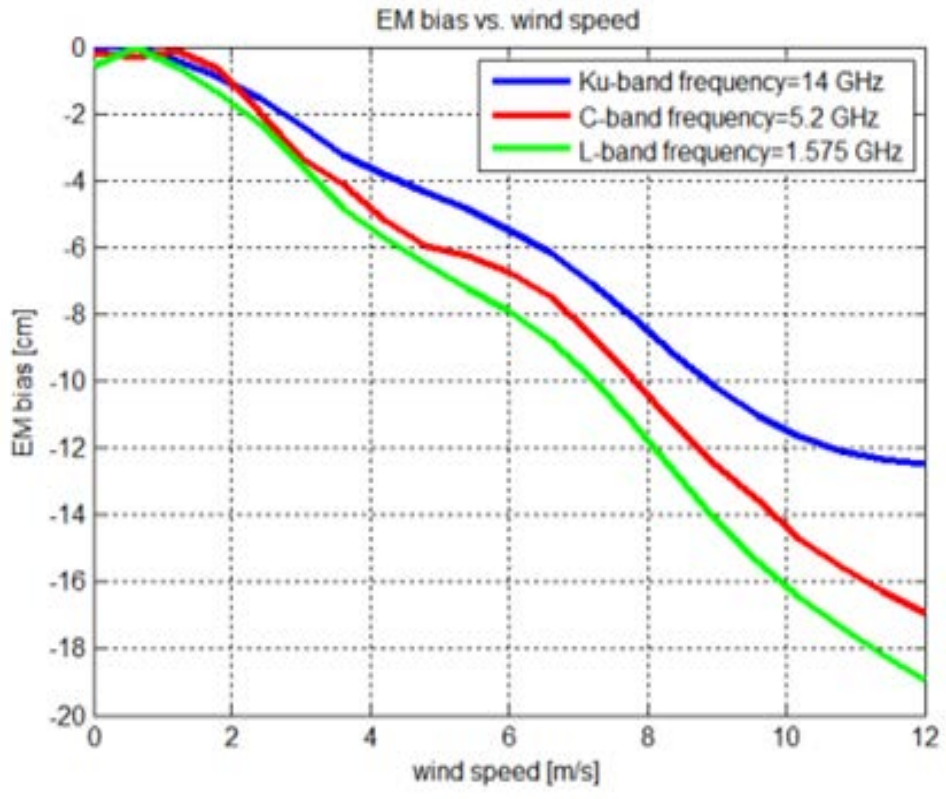

Fig. 7. EM bias vs. significant wave-height at three difference frequencies obtained using the combined model [21], the blue and red ones are the same as in Fig.6, but with the horizontal axis in terms of wind speed [21, Fig. 8, Eqn. 14, 15]. The green one is the extension at L-band. 


\section{Simulation Results and Discussion}

In this Section, results of the EM bias combined model and the numerical method are presented and compared to validate the proposed numerical method, based on the direct evaluation of Eqn. (1). Finally, the effect on the EM bias of some parameters such as: the frequency, incidence/scattering angle, wind direction, sea surface height spectra model, and discretization, are investigated using the proposed numerical method and discussed.

\subsection{Effect of the sea surface height spectrum}

The impact on the EM bias of two both well-known sea surface spectra (Pierson-Moskowitz [25], and Elfouhaily spectra including the spreading function [26]) is examined using the proposed numerical method for an incidence/scattering angle of $25^{\circ}$, and wind directions $0^{\circ}$ and $45^{\circ}$ (Fig. 8). Error bars indicate the 95\% confidence levels.

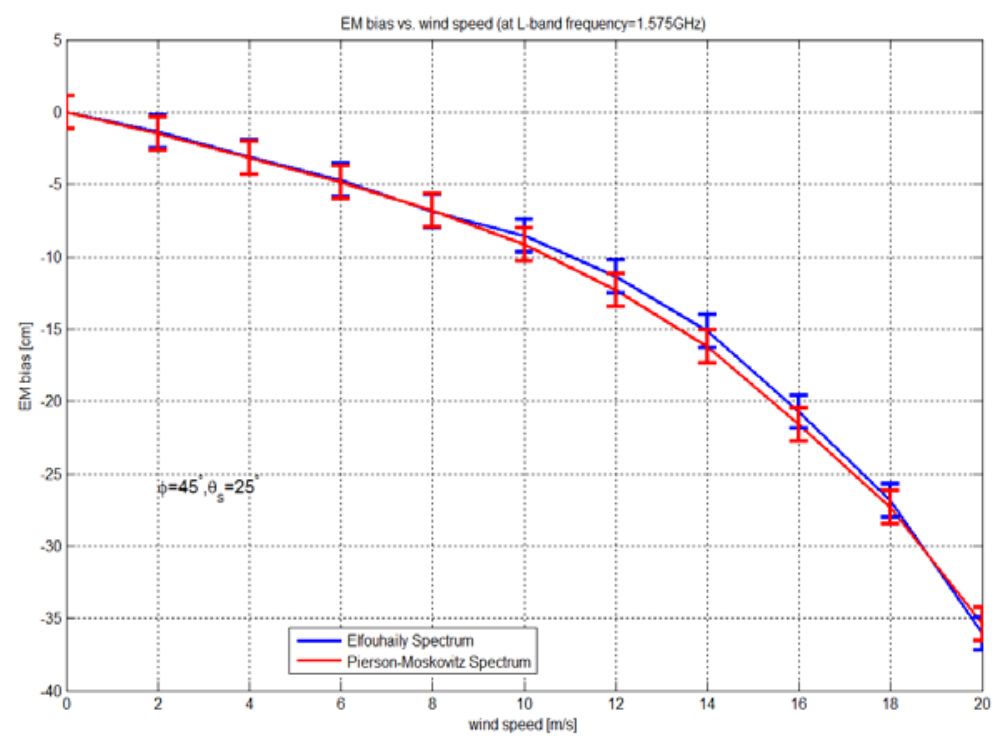

Fig. 8. Comparison of the EM bias (at L-Band) vs. wind speed using the Pierson-Moskowitz [25] and Elfouhaily spectra (facet size $20 \mathrm{~cm}$ ) [26].

It is apparent that the trend is similar, and results are very similar, although for moderate wind speeds there are differences as high as $\sim 2 \mathrm{~cm}$. The results in Fig. 8 indicate that the actual sea surface spectrum is not critical. From now on, the Elfouhaily et al.'s spectrum is used throughout this work. 


\subsection{Impact of surface discretization}

The effects of the short-wave components on the radar cross-section have been investigated experimentally in [48], and they have shown that it is nearly independent on the wind speed at near-nadir incidence angles. However, at larger incidence angles, $\sigma^{0}$ increases with increasing wind speeds.

In order to investigate the impact of the short-wave components on the EM bias, the surface is discretized into smaller facets (10 cm side) and compared to the nominal discretization (20 $\mathrm{cm}$ side). Simulation results at L-band, wind direction of $45^{\circ}$, incidence/scattering angle of $0^{\circ}$ are presented in Fig. 9.

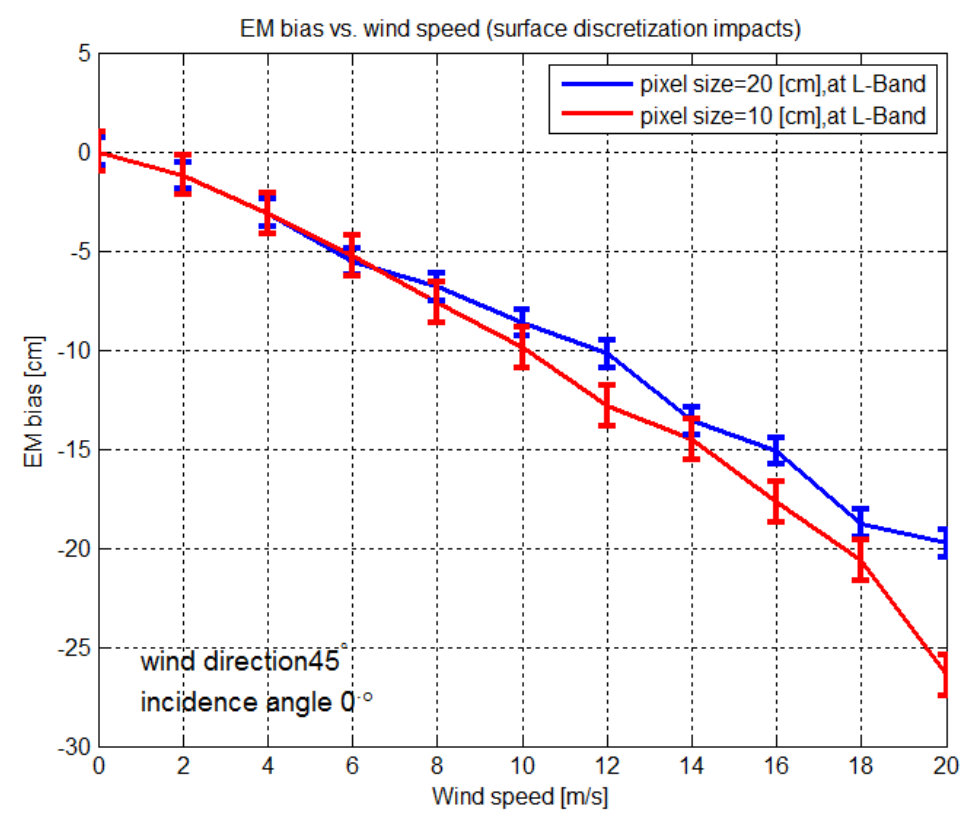

Fig. 9. Comparison of sea surface discretization impact on the EM bias (Elfouhaily et al.'s spectra).

As it can be appreciated, the EM bias difference is negligible for very low wind speeds ( $U_{10} \leq 6 \mathrm{~m} / \mathrm{s}$ ), but it increases with increasing wind speeds, the EM bias being larger with the $10 \mathrm{~cm}$ discretization, than with the $20 \mathrm{~cm}$ one, due to the presence of short wave components: $\sim 2-3 \mathrm{~cm}$ up to $18 \mathrm{~m} / \mathrm{s}$, and $\sim 6 \mathrm{~cm}$ at $20 \mathrm{~m} / \mathrm{s}$. However, the largest contribution to the EM bias is coming from the long waves, in agreement with [21]. 


\subsection{Investigating the incidence angle impact on the EM bias}

In order to investigate the impact of the incidence angle on the EM bias, the EM bias combined model is used to simulate and investigate several incidence/scattering angles $0^{\circ}$, $25^{\circ}$, and $45^{\circ}$ at $\mathrm{Ku}$-band [21, 22] (Fig. 10). As the incidence angle increases, the EM bias $\left(\beta_{E M}\right)$ increases as well, approximately as a cosine function. This is due to the extra transit time $\Delta t$ from transmitter to receiver when the surface is displaced a height $h: \Delta t=2 h / c$. $\cos \theta$. The EM bias is then computed at L-band using our numerical method for the same incidence/scattering angles $0^{\circ}, 2^{\circ}$, and $45^{\circ}$ (Fig. 10). The EM bias is larger at L-band than it is at Ku-band, but the trend with incidence angle is the similar.

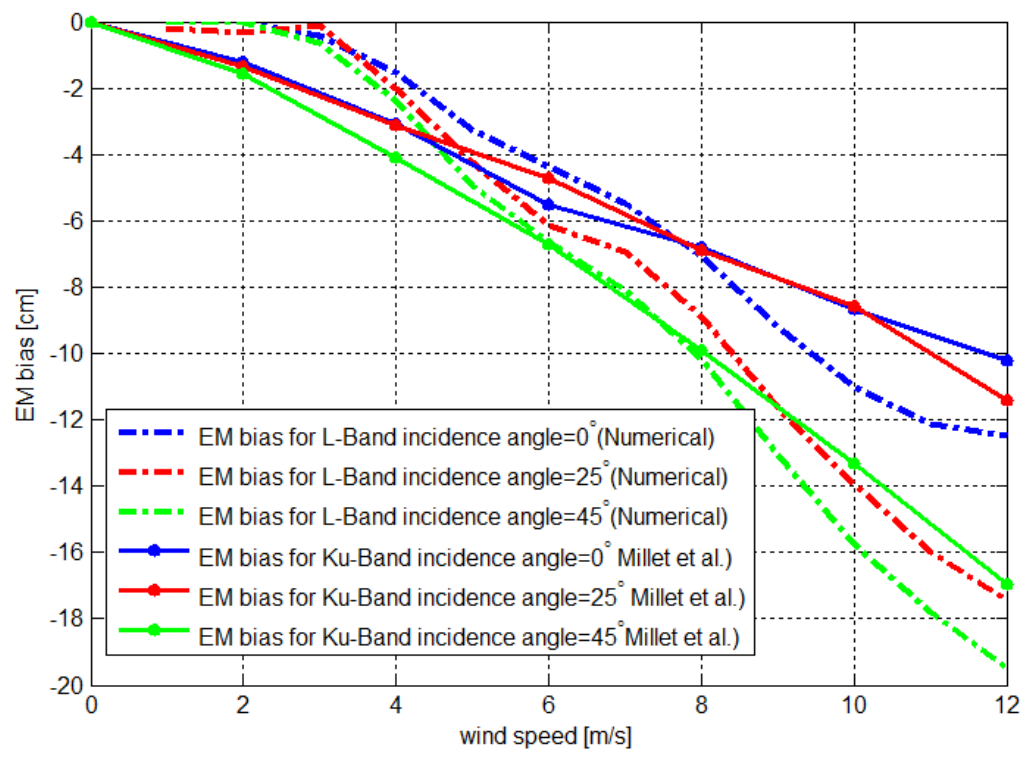

Fig. 10. Comparison of incidence angle impact on the EM bias at L- and Ku-bands using the combined method (Millet et al. method) [21, 22] and the proposed numerical method. Error bars not included for the sake of clarity.

\subsection{Effect of Azimuthal Angle on the EM bias}

Most analytical models do not predict the dependence with the incidence angle, and only the combined model is able to predict it. However, none is able to predict the azimuthal signature that is induced by the angle between the look angle and the wind direction, the dependence on $\sigma^{0}$. In this section, the azimuthal dependence of the EM bias vs. the wind direction is also investigated using the basic definition (Eqn. (1)). Results are shown in Fig. 11 for $\theta_{i, s}=25^{\circ}$, and for three different wind speeds 5, 10, and $15 \mathrm{~m} / \mathrm{s}$. As it can be appreciated, the EM bias 
dependence exhibits a non-negligible azimuthal dependence ( 1.5-2 cm peak-to-peak at 10 $\mathrm{m} / \mathrm{s}$, and $\sim 5 \mathrm{~cm}$ peak-to-peak at $15 \mathrm{~m} / \mathrm{s}$ ). The effect of the wave asymmetry is evident, and it has to be taken into account as well in the error budgets of future GNSS-R altimeters [4].

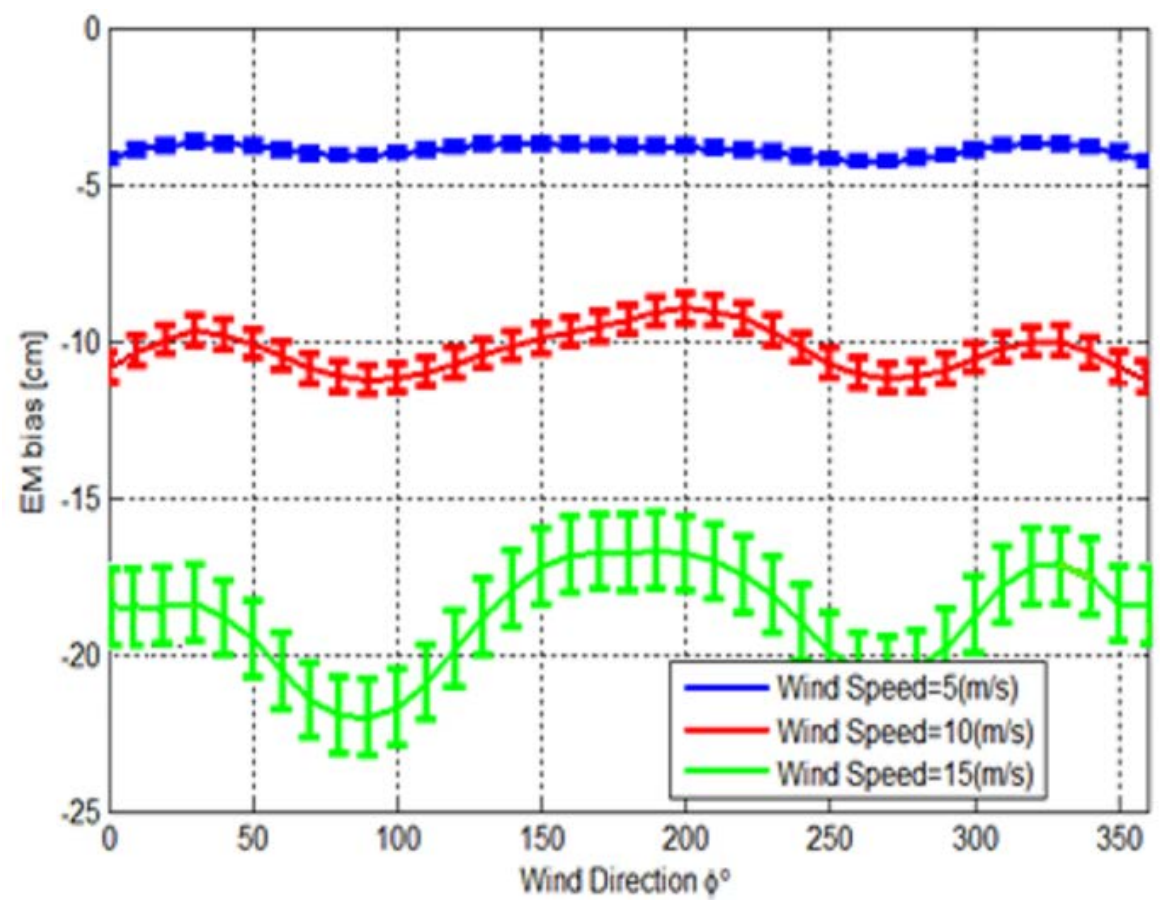

Fig. 11. EM bias vs. wind direction using non-Gaussian sea surface and incidence angle of $\theta_{s}=$ $25^{\circ}$ for $\mathrm{U}_{10}=10,15$ and $20 \mathrm{~m} / \mathrm{s}$.

\section{CONCLUSIONS}

In this work, Millet's EM bias combined model (WNL + MTF) has been implemented including the effect of incidence angle. It has then been validated at C- and Ku-bands with existing experimental data, and then it has been extrapolated at L-band. An efficient numerical approach to compute the EM bias is proposed based on the numerical evaluation of EM bias basic definition, for a realization of the sea surface using the KA PO scattering method. Monte Carlo simulations have been performed to reduce the uncertainty of the estimations. The proposed numerical technique has been validated against Millet's combined model, and it allows to predict the dependence with frequency, incidence/scattering angle, azimuthal angle, and wind speed, which may explain some of the differences found, since analytical models do not account for the azimuthal dependence. 
The impact of the sea surface spectra used is negligible. The impact of the facet size has been evaluated by discretizing the synthetic sea surface at $10 \mathrm{~cm}$ and $20 \mathrm{~cm}$ side. It is found that discretization effects are negligible for low wind speed conditions, but not for high wind speeds, which confirms that long waves have a stronger impact on the EM bias than the short waves, although the short ones do contribute as well.

The general trend is that the EM bias increases with decreasing frequency, increasing incidence angle, and increasing wind speed, and it exhibits an azimuthal angle modulation as

well, that somehow mimics that of $\sigma^{0}$. At L-band, and for $12 \mathrm{~m} / \mathrm{s}$ wind speed, the EM bias at nadir $\left(\theta_{i, s}=0^{\circ}\right)$ can be as high as $13 \mathrm{~cm}$, but at large incidence angles $\left(\theta_{i, s}=45^{\circ}\right)$ it can increase up to $19 \mathrm{~cm}$ (Fig. 10).

These values are very important and will dominate the altimetry error budget of future GNSS$\mathrm{R}$ altimeters [4], unless properly corrected for. The EM bias correction requires a precise knowledge of the geometry (incidence/scattering angle), and the wind speed and azimuthal angle with respect to the incidence plane, as auxiliary information.

\section{ACKNOWLEDGMENTS}

This work has been support by the Spanish National Research project AYA2011-29183-C0201 from the Ministry of Economy and Competitiveness. 


\section{APPENDICES}

\section{A. The off-nadir EM bias}

The off-nadir EM bias model has been estimated using the standard composite scattering model to computing the scattering cross-section [32, 33], It should be emphasized the multiple scattering impact is neglected. Considering the off-nadir EM bias model definition, the following is proposed in [21]:

$$
\epsilon=\frac{\iint \zeta \sigma^{0}\left(\zeta, \theta+\theta_{l}\right) P\left(\zeta, \theta_{l}\right) d \zeta d \theta_{l}}{\iint \sigma^{0}\left(\zeta, \theta+\theta_{l}\right) P\left(\zeta, \theta_{l}\right) d \zeta d \theta_{l}},
$$

where $\zeta$ is the sea surface elevation, $\theta$ is the incident angle, $\theta_{l}$ is the local long wave tilt angle, $\sigma^{0}$ is the scattering cross-section, $P(\zeta, \theta)$ is the joint long wave height tilt distribution, which is estimated by a Gram-Charlier series [15]. The short wave scattering is modeled using PO [21]:

$$
\sigma^{0}(\psi)=\frac{k_{e m}^{2} \cos ^{2} \psi}{4 \pi} \iint e^{i k_{b} x} e^{-\Gamma(1-C(x, y))} d x d y,
$$

where $\psi$ is the local incidence angle of the illuminating electromagnetic wave, $k_{b}=$ $2 k_{e m} \sin \psi$, and $\Gamma=\left(2 k_{e m} h_{s} \cos \psi\right)^{2}, C$ is the correlation function, which is obtained by the Fourier transform of the short wave Power Spectral Density (PSD), $h_{s}^{2}$ is the small wave height variance, and $k_{e m}$ is the electromagnetic wavenumber. Finally, an analytical EM bias is given [21]:

$$
\epsilon(\theta)=-H\left[\gamma(\theta) \cdot v \cdot S+\tau(\theta) \cdot \lambda_{12}\right],
$$

where $\gamma(\theta), \tau(\theta)$ are the short wave coefficients, $S$ is the RMS slope of the long waves, $H$ is the significant wave height, $\lambda_{12}$ is the long wave tilt modulation, $v$ is assumed about 0.7 (it is computed from the RMS wave slope).

In the off-nadir EM bias, based on composite scattering surface, the cut-off wavenumber was not sensitive enough, so the unified Elfouhaily et al.'s spectrum was considered. 


\section{B. Generating a two dimensional time-domain sea surface}

Synthesizing the sea surface includes two main procedures: generation the sea surface spectrum, and converting the spectrum into the time domain. The sea spectrum is generated assuming deep waters (waves are not affected by the seabed), a given intensity, fetch, and direction of wind speed (Pierson-Moskowitz and Elfouhaily spectrum models [25, 26]).

Based on the realistic situation, the sea surface is varying instantaneously. In fact, sea state and time must be involved in the sea surface simulation. To assessment the spectral components of the sea surface, the deep-water transport equation is given [49]:

$$
\frac{\partial}{\partial t} \Psi(\mathrm{k}, \theta ; x, t)+c_{g} . \nabla \Psi(\mathrm{k}, \theta ; x, t)=S_{i}+S_{d}+S_{n l},
$$

where $\mathrm{k}$ is the mechanical wavelength, $\theta$ is the traveling wave direction along the distance $x$, during a time $t, c_{g}$ is the group velocity, and $S_{i}, S_{d}, S_{n l}$ are the wind energy, the dissipation energy, and the dispersive transference energy. The statistical properties of the sea surface have been used to present the roughness spectrum $\Psi(\mathrm{k}, \varphi)$ as the Fourier transform of the autocorrelation function of the sea surface. It includes both the radial spectrum $S(\mathrm{k})$ and the angular spreading function $\Phi(\mathrm{k}, \varphi)$. The angular spreading is approximated using a Fourier series expansion of an even real function [26, 49]:

$$
\begin{gathered}
\Psi(\mathrm{k}, \varphi)=\frac{1}{\mathrm{k}} S(\mathrm{k}) \cdot \Phi(\mathrm{k}, \varphi), \\
\Phi(\mathrm{k}, \varphi)=\frac{1}{2 \pi}\left[1+\sum_{n=1}^{\infty} a_{2 n} \cdot \cos (2 n \varphi)\right], \\
\Phi(\mathrm{k}, \varphi) \approx \frac{1}{2 \pi}\left[1+a_{2} \cos (2 \varphi)\right] .
\end{gathered}
$$

The generation of the time domain sea surface is performed by generating an initial twodimensional random process which are filtered by the directional sea surface spectrum [50]. It means, the random phase filtered by $\Psi(\mathrm{k}, \varphi)$, and the propagation each frequency that is computed according to the deep water dispersion relationship. 


\section{The Physical Optics Model}

First, from the conception that a circular polarized wave (either right or left handed) is a basic combination of two orthogonal linear polarized waves with $90^{\circ}$ phase shift, i.e. H-pol and Vpol [30]:

$$
E_{\text {circ }}=\left(\overrightarrow{E_{V}} \pm j \overrightarrow{E_{H}}\right) \exp (-j \mathbf{k r})
$$

The sea surface scattered field can be found using the KA PO method (Kirchhoff Approximation - Physical Optics). The induced current on the sea surface is:

$$
\overrightarrow{J_{s}}=2 \hat{n} \times \widehat{H},
$$

where $\hat{n}, \widehat{H}$ are the normal vector, and the magnetic field vector of the incidence wave, and $\mathbf{k}=k \cdot \hat{k}$ ( $k$ is the wave vector). Then, the scattering over a finite metallic rectangular plate size $(a \times b)$ is:

$$
\begin{aligned}
& E_{r}^{s} \cong 0 \\
& E_{\theta}^{S}=C \frac{e^{-j k r}}{r}\left\{\cos \theta_{s} \sin \phi_{s}\left[\frac{\sin (X)}{X}\right]\left[\frac{\sin (Y)}{Y}\right]\right\}, \\
& E_{\varphi}^{s}=C \frac{e^{-j k r}}{r}\left\{\cos \theta_{s}\left[\frac{\sin (X)}{X}\right]\left[\frac{\sin (Y)}{Y}\right]\right\}, \\
& X=\frac{k a}{2} \sin \theta_{s} \cos \varphi_{s} \\
& Y=\frac{k b}{2}\left(\sin \theta_{s} \cos \varphi_{s}-\sin \theta_{i}\right) \\
& C=-j \eta \frac{a b k H_{0}}{2 \pi}
\end{aligned}
$$

where $H_{0}$ is the magnitude of the incident magnetic field, and $\eta$ is the intrinsic impedance, $\theta_{s}$ and $\varphi_{s}$ are scattered field elevation and azimuth angles. In the case of a non-metallic surface, the scattered field can be computed using the Impedance Boundary Condition (IBC) as in Eqns. 10-17, multiplying Eqns. 13 and 14 by the Fresnel reflection coefficients [47].

Finally, a $5 \times 5$ microstrip patch array with $\frac{\lambda}{2}$ element spacing is used to simulate the receiving antenna at $5 \mathrm{~km}$ height. 


\section{References}

[1] M. Martin-Neira, “A Passive Reflectometry and Interferometry System (PARIS): application to ocean altimetry,” ESA J, pp. 331-355, 1993.

[2] G. A. Hajj and C. Zuffada, "Theoretical description of a bistatic system for ocean altimetry using the GPS signal,” Radio Sci., vol. 38, no. 5, Oct. 2003.

[3] A. Rius, E. Cardellach, and M. Martín-neira, "Altimetric analysis of the Sea-surface GPS-reflected signals,” IEEE Trans. Geosci. Remote Sens., vol. 48, no. 4, pp. 21192127, 2010.

[4] A. Camps, H. Park, E. Valencia i Domenech, D. Pascual, F. Martin, A. Rius, S. Ribo, J. Benito, A. Andres-Beivide, P. Saameno, G. Staton, M. Martin-Neira, S. Daddio, and P. Willemsen, "Optimization and performance analysis of interferometric GNSS-R altimeters: Application to the PARIS IoD mission,” IEEE J. Sel. Top. Appl. Earth Obs. Remote Sens., vol. 7, no. 5, pp. 1436-1451, 2014.

[5] M. P. Clarizia, C. Gommenginger, M. Di Bisceglie, C. Galdi, and M. A. Srokosz, "Simulation of L-Band Bistatic Returns From the Ocean Surface : A Facet Approach With Application to Ocean GNSS Reflectometry,” IEEE Trans. Geosci. Remote Sens., vol. 50, no. 3, pp. 960-971, 2012.

[6] A. Ghavidel, D. Schiavulli, and A. Camps, "A numerical simulator to evaluate the electromagnetic bias in GNSS-R altimetry,” in 2014 IEEE Int. Geosci. and Rem. Sens. Symposium, pp. 4066-4069, 13-18 July 2014

[7] B. S. Yaplee, A. Shapiro, D. L. Hammond, B. D. Au, and E. A. Uliana, "Nanosecond radar observations of the ocean surface from a stable platform," IEEE Trans. Geosci. Electron., vol. 9, no. 3, 1971.

[8] T. Elfouhaily, D. R. Thompson, B. Chapron, and D. Vandemark, "Improved electromagnetic bias theory,” J. Geophys. Res. Ocean., vol. 105, pp. 1299-1310, 2000.

[9] T. Elfouhaily, D. R. Thompson, D. Vandemark, and B. Chapron, "Higher-order hydrodynamic modulation: theory and applications for ocean waves," Proc. R. Soc. Lond. A vol. 457, no. 2015, pp. 2585-2608, Nov. 2001.

[10] F. C. Jackson, “The reflection of impulses from a nonlinear random sea,” J. Geophys. Res. Ocean., vol. 84, pp. 4939-4943, 1979.

[11] M. S. Longuet-Higgins, "The effect of non-linearities on statistical distributions in the theory of sea waves,” J. Fluid Mech., vol. 17, no. 1955, pp. 459-480, 1963.

[12] D. E. Barrick, "Determination of mean sea surface position and sea state from the radar return of a short-pulse satellite altimeter, sea surface topography from Space," NOAA Sea Surf. Topogr. from Sp., vol. 1, p. 19, 1972.

[13] W. J. Pierson and E. Mehr, "Average return pulse form and bias for the S193 radar altimeter on Skylab as a function of wave conditions, in The Use of Artificial Satellites for Geodesy (eds S. W. Henriksen, A. Mancini and B. H. Chovitz), American Geophysical Union, Washington, D. C.. doi: 10.1029/GM015p0217, vol. 15, 1972.

[14] G. Brown, "The average impulse response of a rough surface and its applications,” IEEE Trans. Antennas Propag., vol. 25, no. 1, pp. 67-74, Jan. 1977.

[15] M. A. Srokosz, "On the joint distribution of surface elevation and slopes for a nonlinear random sea, with an application to radar altimetry,” J. Geophys. Res. Ocean., vol. 91 C1, pp. 995-1006, 1986.

[16] R. E. Glazman, A. Fabrikant, and M. A. Srokosz, "Numerical analysis of the sea state bias for satellite altimetry,” J. Geophys. Res. Ocean., vol. 101, pp. 3789-3799, 1996.

[17] T. Elfouhaily, D. Thompson, D. Vandemark, and B. Chapron, "Weakly nonlinear theory and sea state bias estimations,” Journal of Geophysical Research, 1999. . 
[18] W. Alpers and K. Hasselmann, "The two-frequency microwave technique for measuring ocean-wave spectra from an airplane or satellite," Boundary-Layer Meteorol., vol. 13, pp. 215-230, 1978.

[19] E. J. Walsh, F. C. Jackson, D. E. Hines, C. Piazza, L. G. Hevizi, D. J. Mclaughlin, R. E. McIntosh, R. N. Swift, J. F. Scott, J. K. Yungel, and E. B. Frederick, "Frequency dependence of electromagnetic bias in radar altimeter sea surface range measurements,” J. Geophys. Res. Ocean., vol. 96, issue C11, pp. 20571-20583, 1991.

[20] L. G. Hevizi, E. J. Walsh, R. E. McIntosh, D. Vandemark, D. E. Hines, R. N. Swift, and J. F. Scott, "Electromagnetic bias in sea surface range measurements at frequencies of the TOPEX/POSEIDON satellite,” IEEE Trans. Geosci. Remote Sens., vol. 31, pp. 376-388, 1993.

[21] F. W. Millet, K. F. Warnick, and D. V. Arnold, "Electromagnetic bias at off-nadir incidence angles,” J. Geophys. Res., vol. 110, no. C9, pp. 1-13, 2005.

[22] F. W. Millet, K. F. Warnick, J. R. Nagel, and D. V Arnold, "Physical optics-based electromagnetic bias theory with surface height - slope cross-correlation and hydrodynamic modulation,” IEEE Trans. Geosci. Remote Sens., vol. 44, no. 6, pp. 1470-1483, 2006.

[23] D. B. Creamer, F. Henyey, R. Schult, and J. Wright, "Improved linear representation of ocean surface waves,” J. Fluid Mech., vol. 205, no. 1, pp. 135-161, Apr. 1989.

[24] P. Naenna and J. T. Johnson, "A Monte Carlo study of altimeter pulse returns and the electromagnetic bias,” IEEE Trans. Geosci. Remote Sens., vol. 48, no. 8, pp. 32183224, 2010.

[25] W. J. Pierson and L. Moskowitz, “A proposed spectral form for fully developed wind seas based on the similarity theory of S. A. Kitaigorodskii,” J. Geophys. Res., vol. 69, pp. 5181-5190, 1964.

[26] T. Elfouhaily, B. Chapron, K. Katsaros, and D. Vandemark, “A unified directional spectrum for long and short wind-driven waves,” J. Geophys. Res., vol. 102, no. C7, p. 15781, 1997.

[27] M. S. Longuet-Higgins, D. E. Cartwrigth, and N. D. Smith, "Observation of the directional spectrum of sea waves using the motions of a flotation buoy, in ocean wave spectra,” Prentice-Hall, Englewood Cliffs, NJ, pp. 111-136, 1963.

[28] V. I. Tatarskii and V. V. Tatarskii, "Statistical non-Gaussian model of sea surface with anisotropic spectrum for wave scattering theory. Part I,” Prog. Electromagn. Res., vol. PIER 22, pp. 259-291, 1999.

[29] V. U. Zavorotny and A. G. Voronovich, "Scattering of GPS signals from the ocean with wind remote sensing application,” IEEE Trans. Geosci. Remote Sens., vol. 38, no. 2, pp. 951-964, Mar. 2000.

[30] C. A. Balanis, Advanced engineering electromagnetics. John Wiley \& Sons, 1989, pp. 586-594.

[31] T. Elfouhaily, D. R. Thompson, B. Chapron, D. Vandemark, "Improved electromagnetic bias theory: Inclusion of hydrodynamic modulations," J. Geophys. Res. Ocean., vol. 106, pp. 4655-4664, 2001.

[32] K. F. Warnick and D. V. Arnold, "Generalization of the geometrical-optics scattering limit for a rough conducting surface,” J. Opt. Soc. Am. A, JOSAA, vol. 15, no. 9, pp. 2355-2361, Sep. 1998.

[33] D. V. Arnold, W. K. Melville, R. H. Stewart, J. A. Kong, W. C. Keller, and E. Lamarre, "Measurements of electromagnetic bias at Ku and C bands," J. Geophys. Res., vol. 100, pp. 969-980, 1995. 
[34] A. K. Fung and and H. Chan, "Backscattering o f waves by composite by rough surfaces,” IEEE Trans. Antennas Propag., no. 5, pp. 590-597, 1969.

[35] O. Brown, "Measures of Shape : Skewness and Kurtosis," 2014. [Online]. Available: http://www.tc3.edu/instruct/sbrown/stat/shape.htm.

[36] G. Brown, "Backscattering from a Gaussian-distributed perfectly conducting rough surface,” IEEE Trans. Antennas Propag., vol. 26, no. 3, pp. 472-482, May 1978.

[37] D. E. Barrick and W. H. Peake, "A review of scattering from surfaces with different roughness scales,” Radio Sci., vol. 3, pp. 865-868, 1968.

[38] J. T. Johnson, J. V. Toporkov, and G. S. Brown, “A numerical study of backscattering from time-evolving sea surfaces: comparison of hydrodynamic models," IEEE Trans. on Geosci. and Rem. Sens., vol.39, no.11, pp.2411-2420, Nov. 2001.

[39] N. I. Nickolaev, O. I. Yordanov, and M. A. Mickalev, "Radar backscattering from non-Gaussian sea surface," Geoscience and Remote Sensing Symposium, 1991. IGARSS '91. Remote Sensing: Global Monitoring for Earth Management., International , vol.3, pp.1275-1278, 3-6 Jun 1991.

[40] N. I. Nickolaev, O. I. Yordanov, and M. a. Michalev, "Non-Gaussian effects in the two-scale model for rough surface scattering,” J. Geophys. Res., vol. 97, no. C10, pp. 15617-15624, 1992.

[41] V.I. Tatarskii and V.V. Tatarskii, "Statistical non-Gaussian model of sea surface with anisotropic spectrum for wave scattering theory. Part I . Chapter 12 (pages 259-291) in Progress in Electromagnetics Research, PIER 22, J. A. Kong, Chief Editor, EMW Publishing, Cambridge, Massachusetts, USA, 1999.

[42] V. V. Tatarskii and V. I. Tatarskii, "Non-Gaussian statistical model of the ocean surface for wave-scattering theories," Waves in Random Media, vol. 6, pp. 419-435, 1996.

[43] C. Cox and W. Munk, "Statistics of the sea surface derived from sun glitter," J. Mar. Res., vol. 13, pp. 198-227, 1954.

[44] C. Cox and W. Munk, "Measurement of the roughness of the sea surface from photographs of the sun’s glitter,” J. Opt. Soc. Am., vol. 11, pp. 838-850, 1954.

[45] M. S. Longuet-Higgins, "The statistical geometry of random surfaces,” Proc. Symp. Appl. Math. Hydrodyn. Instab. Amer. Math. Soc, pp. 105-143, 1962.

[46] N. Huang and S. R. Long, "An experimental study of the surface elevation probability distribution and statistics of wind-generated waves," J. fluid Mech, vol. 101, pp. 179220, 1980.

[47] J. M. Rius, M. Ferrando, and L. Jofre, "GRECO: graphical electromagnetic computing for RCS prediction in real time,” IEEE Antennas Propag. Mag., vol. 35, no. 2, pp. 717, Apr. 1993.

[48] D. J. Donohue, H. Ku, D. R. Thompson, and J. Sadowsky, "Direct numerical simulation of electromagnetic rough surface and sea scattering by an improved banded matrix iterative method,” Johns Hopkins APL Tech. Dig. (Applied Phys. Lab., vol. 18, no. 2, pp. 204-215, 1997.

[49] K. Hasselmann, "On the non-linear energy transfer in a gravity-wave spectrum Part 1. General theory,” J. Fluid Mech., vol. 12, no. 4, pp. 481-500, 1962.

[50] G. Mastin, P. Watterberg, and J. Mareda, "Fourier synthesis of ocean scenes," IEEE Comput. Graph. Appl., vol. 7, no. 3, pp. 16-23, Mar. 1987. 